\section{Qualität des Naturdargebotes in der Landnutzung. Soziale und ökonomische Bewertung als Instrument der Entschei- dungsunterstützung ${ }^{1}$}

\author{
von Annette Henn und Renate Patz, Fach- \\ hochschule Merseburg
}

Im Mittelpunkt des vom UFZ Umweltforschungszentrum Leipzig-Halle $\mathrm{GmbH}$ geförderten Projektes an der Fachhochschule Merseburg steht die Beantwortung der forschungsleitenden Fragestellung, wie viel und welche Natur qualitativ und quantitativ aus ökologischer, ökonomischer und sozialer Perspektive in welchen räumlichen Positionen in urbanen Landschaften notwendig, sinnvoll und unter welchen Bedingungen möglich ist. Gerade in von Strukturwandel betroffenen Regionen genießt die ökonomische Strukturentwicklung in der Planung immer noch die oberste Priorität. Eine der Nachhaltigkeit verpflichtete räumliche Planung und Entwicklung muss jedoch sowohl die Erhaltung und den Schutz raumspezifisch vorhandener natürlicher und naturnaher Ressourcen berücksichtigen, also des vorhandenen Naturkapitals, wie auch eine angemessene wirtschaftliche und sozial gesicherte Entwicklung. Eine ihrer grundlegenden Aufgaben ist damit die Sicherung und Entwicklung von Grün- und Freiflächen bzw. naturräumlichen Einheiten, einschließlich ihrer ökologischen Funktionalität, ökonomischen Tragfähigkeit und der damit verbundenen steigenden Lebensqualität der gesamten Region. Im vorliegenden Beitrag wird ein neuer Ansatz zur Entscheidungsunterstützung vorgestellt, der es ermöglicht, die Belange sämtlicher Interessenvertreter abzubilden und im Planungsprozess adäquat zu berücksichtigen.

\section{Vorüberlegungen}

Ein zunehmender Verbrauch naturräumlicher Ressourcen ist eine Folge des Akteursverhaltens. Eine Verständigung über mögliche Interessenkonflikte muss bei den individuellen Bedürfnissen von Akteuren und daraus abgeleiteten gesamtgesellschaftlichen Ansprüchen ansetzen. Generell stellen die zu betrachtenden Akteure die an gesellschaftlich bedeutsamen Meinungsund Willensbildungsprozessen beteiligten Handlungsträger sowohl auf institutioneller wie auch auf individueller Ebene dar. Im Rahmen einer Untersuchung zu Partizipation an und Akzeptanz von nachhaltiger Regionalentwicklung erscheint es sinnvoll, über die institutionellen Handlungsträger (vgl. Jänike 1995) hinaus jene Akteure zu erfassen, die mittel- oder unmittelbar von umweltpolitischen Entscheidungen betroffen sind, also gewissermaßen Begrenzungen ihres individuellen Handlungsspielraumes durch planerische Eingriffe hinzunehmen haben. Bei einer Rollenzuweisung ist zwischen konkret abzugrenzenden Untersuchungsräumen und in Bezug auf bestimmte Entscheidungen in den Räumen von unterschiedlichen Akteurskonstellationen auszugehen. Weiterhin sind die Interessenlagen der $\mathrm{zu}$ betrachtenden Akteure sehr heterogen, so dass keine generellen, theoriegeleiteten Unterstellungen der Handlungsmotivation eines Akteurs akzeptiert werden können. Eine Analyse der Akteurskonstellationen in den Untersuchungsräumen muss sich an den Charakteristika der Akteure der Planung und ihren jeweiligen Zielen orientieren. Als Charakterisierungsmerkmale gelten allgemein Interessen- und Machtlagen sowie Wirtschafts-, Rechts- und Informationslagen. Von Bedeutung sind darüber hinaus die Konfiguration der Akteure und deren Veränderung im Zeitverlauf.

Unterstellt man, dass jede Planung eine Veränderung der Umweltbedingungen zur Folge hat, also jegliche Maßnahme einen Einschnitt in den Naturraum oder das Naturkapital ${ }^{2}$ bewirkt, dann sind es in erster Linie jene Akteure, die rechtliche, wirtschaftliche und politische Instrumentarien zur Hand haben, die als Verursacher und/oder Auftraggeber eines Ressourcenverbrauchs auftreten. Von diesen staatlichen bzw. nichtstaatlichen institutionellen Eingriffen sind wiederum andere Akteure in ihrem individuellen Handlungsspielraum eingeschränkt.

\section{Methodik}

Aufgrund dieser Vorüberlegungen und der allgemeinen Forderung, Akteure verstärkt in nachhaltige Politikentwicklung einzubinden, stellte sich zunächst die Frage, welche Methodik die Akteurskonstellationen geeignet abbildet und 
Entscheidungsunterstützung in der Ableitung von Strategieempfehlungen für nachhaltige Planung liefert. Im Rahmen unserer Untersuchungen haben wir geprüft, inwieweit der Einsatz von Marketinginstrumenten, die bereits im Freizeit-Marketing, insbesondere im Destinationsmanagement (vgl. Agricola 2001) Anwendung finden, zur Problemlösung beitragen können. Zentrales Ziel dieser Ansätze ist es, einen Markt herzustellen, zu sichern und einem Konsumentenkreis vorzuführen, um ihn zum Besuch anzuregen. Gerade diese Zielstellung soll genutzt werden, um die betroffenen sowie beteiligten Akteure zum Handeln im Sinne einer nachhaltigen Entwicklung zu motivieren.

Überträgt man also den Ansatz auf einen Markt im Sinne eines ökonomisch-ökologischen Systems (nach Messerli und Messerli 1978), welches sich aus unterschiedlichen räumlichen Elementen und Funktionen zusammensetzt, lässt sich postulieren:

1. Institutionelle Eingriffe wirken gestaltend auf das Angebot von Lebens-, Natur- und Landschaftsqualität. Die maßgebenden Akteure lassen sich somit als Anbieter oder Gestalter beschreiben.

2. Eingriffe reglementieren den Handlungsspielraum weiterer Akteure, womit deren Aktivitäten in den Räumen mittel- bzw. unmittelbar von den Gestaltungsansätzen beeinflusst werden. Es handelt sich dabei also um Akteure der Nachfrage bzw. Nutzer.

Folglich existieren auch in Bezug auf die oben aufgeworfene Fragestellung Angebots- und Nachfragebeziehungen. Ein solcher akteursbezogener Lösungsweg zur Beantwortung der forschungsleitenden Fragestellung besitzt den Vorteil, dass organisierte Handlungsträger bzw. Gestalter als Impulsgeber und ausführendes Organ von Entwicklungsoptionen in der Regional- und Landschaftsplanung in Verbindung $\mathrm{zu}$ den eigentlich Betroffenen gesetzt werden, deren Akzeptanz für umweltrelevante Entscheidungen zu schaffen ist. Akzeptanz kann wiederum nur geschaffen werden, wenn die Bedürfnisse dieser Betroffenen bekannt sind. Damit ist eine Beteiligung von Nutzern und Betroffenen im Sinne von Zielgruppen gewährleistet und die wesentliche Grundvoraussetzung für Nachhaltigkeit erfüllt. ${ }^{3}$
Auf Basis der Definition unterschiedlicher Akteursgruppen wird folglich untersucht, welche Qualitätsanforderungen relevante Zielgruppen an das Produkt „Landschaft“ im urbanen Raum stellen. In diesem Sinne können sich „Anbieter von Landschaft" in der Produktgestaltung nur auf aus Sicht der Nachfrage wahrnehmbare oder nutzbare Produkteigenschaften konzentrieren, die jedoch den Erhalt essentieller natürlicher und naturnaher Ressourcen gewährleisten müssen.

Das Nachfrageverhalten wurde aus vorliegenden Belegquellen in Form einer Sekundäranalyse abgeleitet. Zielstellung ist die Identifikation einer gesamtgesellschaftlichen Bewertung aus individuellen Werturteilen von Nutzern und Betroffenen im Sinne von use und non-usevalues (vgl. u. a. Bateman 1995). Diese werden als Wahrnehmungs- und Nutzungspräferenzen dargestellt und $\mathrm{zu}$ Anforderungsprofilen von (potenziell) nachfragenden Akteuren an Naturangebote in suburbanen Räumen formiert.

Unsere Analyse fokussiert auf eine Erhebung des Anbieterverhaltens. Zentral ist dabei die Fragestellung, welche Motive Gestalter haben, ein ökologisch relevantes Naturangebot in den Untersuchungsräumen unter Berücksichtigung der Dimensionen Wirtschaftlichkeit und Soziales bereitzustellen. Darauf aufbauend wird auch für die Gestaltungsoptionen des Angebotes eine Präferenzrangordnung in Abhängigkeit der zur Verfügung stehenden Budgets zur Regionalentwicklung erstellt. Das heißt, auch für die Anbieter ist ein nach deren Präferenzen gewichtetes Anforderungsprofil zu entwickeln.

Um die Gegenüberstellung des Anbieterund Nachfragerverhaltens zu ermöglichen, wird ein Modellierungsansatz in Anlehnung an eine Bilanzierung zur Erhebung von Angebotsüberschüssen bzw. -defiziten vorgeschlagen. Die Grundlage dafür bilden die zuvor erhobenen Anforderungsprofile im Sinne von Angebotsund Nachfragefunktion.

Gefordert wird eine weitgehende Erfüllung der Anforderungen der Nutzer durch ein bestimmtes Angebot. Im Projektsinne bedeutet das, eine Optimierungslösung zwischen beiden Funktionen herbeizuführen. Da jedoch die Kriterien der Anforderungsprofile nicht unendlich substituierbar sind, zudem Fragen zur Ethik eines umsichtigen Naturumgangs ${ }^{4}$ berührt werden, kann eine solche Modellierung 
nicht zugelassen werden. Vielmehr geht es um die Darstellung einer Näherungslösung, die sowohl Präferenzen der Anbieter und Nachfrager, Kriterienkonkurrenzen beider Akteursgruppen untereinander sowie den Schutz und Erhalt essentieller natürlicher Ressourcen im Raum berücksichtigt. ${ }^{5}$

\section{Der Akteurs-Raum-Bezug}

Der bisherigen Argumentation folgend kann eine nachhaltige Entwicklung von Räumen nur im Kontext individuell und gesellschaftlich gewünschter und präferierter Zielsetzungen gesehen werden. Als anzustrebende gesellschaftliche Zielfunktion ist die Bewahrung der natürlichen Lebensgrundlagen, also des natürlichen Kapitals, einzusetzen. Damit soll sichergestellt werden, dass nicht ausschließlich kurzfristige gesellschaftliche Zeitpräferenzen und ökonomische Effizienz die Entwicklung des Raumes bestimmen. Die Frage nach Präferenzen der Akteure wie auch nach zu bewahrenden natürlichen Lebensgrundlagen kann nur anhand der Spezifik ausgewählter Untersuchungsräume beantwortet werden. Für diese Untersuchungsräume stehen als Bewertungsgrundlagen Flächengröße sowie prozentuale Anteile einzelner Flächennutzungsformen mit ihren prägenden landschaftlichen Attributen zur Verfügung.

Zielgröße der Bewertung ist die Erholungsnutzung und Erholungseignung der Räume als exemplarische Form der Nutzung. Gerade für Erholungssuchende sind weitgehend alle räumlichen Potenziale und deren Wechselwirkung mit dem natürlichen Kapital von Bedeutung, so dass aus deren Sicht eine breite Palette von Aussagen zur geforderten Ausstattung des Raumes mit spezifischen landschaftlichen Parametern erwartet werden konnte. Deren Anforderungen können sowohl dem realen Zustand mit seiner Bandbreite und Komplexität landschaftlicher Eigenschaften als auch den Entwicklungszielen für den Raum bilanziell gegenübergestellt werden. Normative Vorgaben im Sinne von Umweltqualitätszielen auf Basis naturwissenschaftlich-ökologischer Untersuchungen definieren die unantastbaren essentiellen und nicht durch andere landschaftliche Elemente substituierbaren Ressourcen als wesentliches Zukunftspotenzial gerade von stadtnahen Räumen, die einen wesentlichen
Erfolgsfaktor der Erholungsnutzung ausmachen. ${ }^{6}$ Diese Festlegungen sind im Sinne nachhaltiger Planung und Entwicklung der Analyse von Nutzungsformen und -beschränkungen zugrunde zu legen.

Ausgehend davon, dass Umweltpräferenzen der Nachfrage in Abhängigkeit von der wirtschaftlichen Entwicklung, der bestehenden Wirtschaftsstruktur, dem Bildungsniveau, der religiös-kulturellen Tradition, der Rechtsordnung etc. divergieren (vgl. Klemmer 1995), waren durch Nutzung geprägte typische Räume auszuwählen. Raumbezogen ließ sich daraus eine unterschiedliche Bereitschaft zum umweltund strukturpolitischen Handeln ableiten, die wiederum unterschiedliche intraregionale $\mathrm{Ab}$ stimmungsproblematiken mit sich bringt. Für die Untersuchung wurden unter Berücksichtigung administrativer Grenzen und damit in Abhängigkeit aktiver Einflussnahme bestimmter Akteursgruppen die folgenden Räume in der Stadt-Umland-Region Halle-Leipzig betrachtet:

- Bergbaufolgelandschaft

Die Aufnahme einer Bergbaufolgelandschaft in die Betrachtung verspricht gerade für die Umsetzung von Nachhaltigkeitskonzepten große Erfolgsaussichten, da sich diese Landschaften im komplexen Umbruch befinden, also jegliche Elemente des oben angesprochenen Potenzialgefüges in Frage gestellt werden, wenn es um die Revitalisierung des Raumes geht. Die komplexe Veränderung kann nach dem vorherrschenden Wertesystem erfolgen. Hier besteht andererseits aber die größte Chance, eine nachhaltige Entwicklung des Raumes zu schaffen. Von besonderer Bedeutung für eine nachhaltige Entwicklung sind Entwicklungsziele zur Revitalisierung und zum Flächenrecycling ehemals bergbaulich sowie industriell genutzter Gebiete. Aus ökologischer Sicht scheinen vor allem geplante Schutzgebietsausweisungen interessant.

- Auenlandschaft

Die Auenlandschaft stellt im stadtnahen Raum den Typus einer naturnahen Landschaft dar. Sie ist sehr kleinräumig differenziert und strukturiert, was sich stark in der naturräumlichen Differenzierung widerspiegelt. Obwohl auch die Auenlandschaft bereits anthropogen überformt ist, ist sie durch eine geringere Nutzungsintensität als andere 
Landschaftstypen geprägt. Dadurch weist sie aus ökologischer Sicht die wertvollsten Landschaftselemente auf, was gerade für die Erholungsnutzung von besonderem Interesse ist. Die Erhebung hier fokussiert somit auf Wechselwirkungen, die durch vornehmlich ökonomische Entscheidungen mit bestehendem Naturkapital auftreten.

\section{- Agrarlandschaft}

Als dritter Raum wurde eine Landschaft aufgenommen, in der jeglicher städtische Charakter ausgeklammert werden kann. Das sind typische Landschaften, die sich fast ausschließlich auf landwirtschaftliche Nutzungsformen beschränken, also offene Agrarlandschaften. Im Kontext der Erholungseignung und -nutzung sind gerade jene Ausschnitte zu betrachten, die zum inneren Ring des Stadtumlandes gehören und damit insbesondere für die Naherholung bedeutsam sind.

Es handelt sich bei diesen drei zu betrachtenden Untersuchungsräumen um historisch gänzlich gegensätzlich geprägte Kulturlandschaften, die jedoch jeweils eine typische Landschaft im städtischen Umland der Region Halle-Leipzig repräsentieren. Durch die Wahl der Untersu- des Einflusses der Nutzung und Gestaltung abbildbar.

\section{Ausgewählte Ergebnisse}

Ein Schwerpunkt unserer Arbeit lag auf der Identifikation der am Planungsprozess maßgeblich beteiligten Akteure in den drei Untersuchungsräumen in Abhängigkeit von ihrer Sanktionsmacht bzw. ihrem Handlungspotenzial. ${ }^{7}$ Belegt wurde, dass auf Grund der festgestellten Akteursbeziehungen der Raum, insbesondere der Kulturraum, Ergebnis menschlichen Handelns ist. Zulässig ist daher die Übertragung mikroökonomischer Instrumentarien, insbesondere von Stakeholder-Modellen, auf die Ebene des Raumes im Sinne erweiterter StakeholderÖkonomien (vgl. Freimann 1998). Als Akteure der Nachfrage von Freizeit- und Erholungsangeboten oder auch Zielgruppe der Planung sind konkret Bewohner, Naherholungssuchende und Touristen definiert worden. Die Akteure der Gestaltung lassen sich - wie in Abbildung 1 dargestellt - nach ihren individuellen Zielsetzungen im Planungsprozess systematisieren:

Die einzelnen Akteursgruppen wurden im Rahmen einer empirischen Erhebung zur Be-

Abb. 1: Akteure des Angebotes (gem. eigener Erhebung im Jahr 2001)

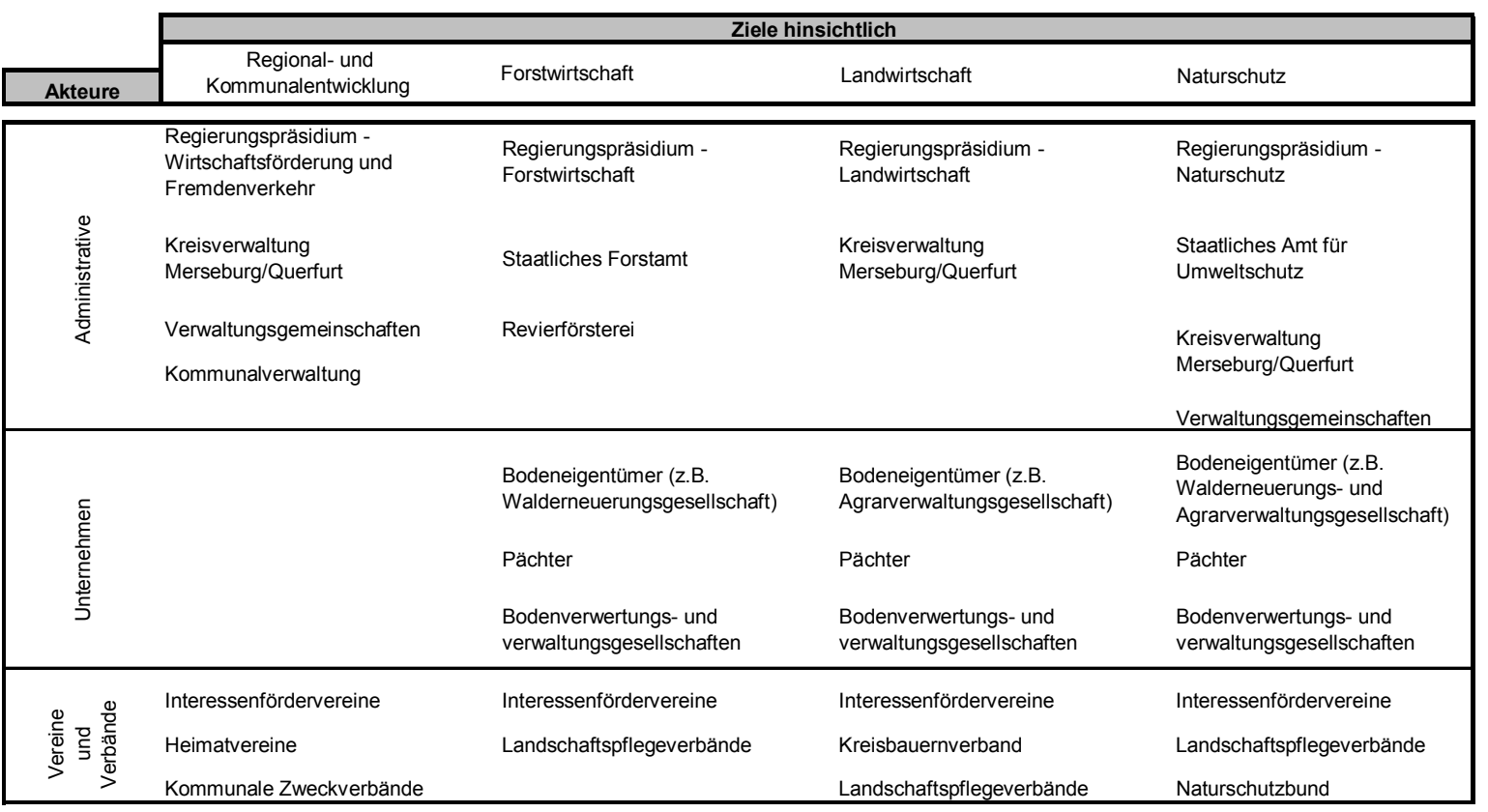


derungen und Entwicklungszielen in Bezug auf die Gestaltung der ausgewählten Untersuchungsräume befragt. Festgestellt wurde, dass die Anforderungen und Zielsetzungen der Akteure die Aktionsparameter zur Gestaltung der Räume stark reglementieren. Im Ergebnis der Untersuchungen konnten folgende Aussagen getroffen werden: Nachfrage und Nutzungen von Natur und Landschaft bilden sich aus Wünschen und Bedürfnissen der Bewohner und Erholungssuchenden heraus. Dabei können Ansprüche auf einem breiten Nachfragekonsens beruhen bzw. sehr spezifisch mit bestimmten Freizeitaktivitäten und Nutzungen verbunden sein. Um Anforderungen der Nachfrage zu erfassen, sind wahrnehmungsorientierte Ansprüche in Bezug auf das Landschaftsbild und aktivitätsorientierte Ansprüche zur Nutzungslandschaft zu berücksichtigen. Beide Ebenen wahrnehmungs- und aktivitätsorientierter Bedürfnisse wurden $\mathrm{zu}$ einem Profil aggregiert, um eine ganzheitliche Aussage über das Nachfrageverhalten in Bezug auf Landschaftspräferenzen, -ausstattungspräferenzen, Häufigkeiten und Frequenzen der Nutzung zu treffen und damit Referenzwerte für die Entwicklung eines adäquaten Angebotes im Raum bereitzustellen.

Die durchgeführte Analyse der Bedürfnisstrukturen der im Raum relevanten Akteursgruppen bildete die Basis für die bilanzierende Gegenüberstellung von Anforderungsprofilen zwischen Angebot und Nachfrage zur Identifikation von Angebotsüberschüssen bzw. -defiziten. Parallel dazu konnten Interaktionen und Kriterienkonkurrenzen zwischen den Beteiligten deutlich gemacht werden. Bilanz wurde jedoch nicht nur zwischen den häufig konfligierenden Zielsetzungen der Akteure gezogen. Vielmehr sollte auch die Frage, wie Bedürfnisse und Anforderungen der Akteure durch die Beschaffenheit des Raumes erfüllt sind, beantwortet werden. Grundlage dafür bildet die Systematisierung erholungs- und freizeitrelevanter räumlicher Parameter in Form eines Landschaftsinventars. Daran schloss sich eine Gegenüberstellung der Entwicklungsziele zur Erreichung des Plan-Zustandes an. Realer und angestrebter Zustand des Raumes wurden anhand des nachfolgend abgebildeten Landschaftsinventars bewertet (vgl. Abb. 2). Gefragt wurde nach der Bedeutsamkeit der hier erfassten landschaftlichen Attribute für die Wahrnehmung und Nutzbarkeit des Raumes durch die betrachteten Akteursgruppen.

\section{Abb. 2: Landschaftsinventar ${ }^{8}$}

\begin{tabular}{|c|c|}
\hline & yenschaften \\
\hline 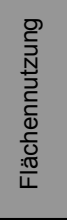 & $\begin{array}{l}\text { Anteil der landwirtschaftlichen Nutzflächen } \\
\text { Anteil der Waldfläche } \\
\text { Anteil der Gewässerfläche } \\
\text { Anteil der Siedlungs- und Verkehrsflächen } \\
\text { Anteil der Erholungsfläche } \\
\text { Vielfalt des Flächennutzungsgefüges }\end{array}$ \\
\hline 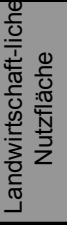 & $\begin{array}{l}\text { Vielfalt nach Schlagflächengröße } \\
\text { Gliederung durch Gehölze } \\
\text { Ackerrandstreifen } \\
\text { Vielfalt der Nutzung } \\
\text { Grünlandanteil } \\
\text { Dichte des Feldwegenetzes }\end{array}$ \\
\hline $\begin{array}{l}\frac{5}{0} \\
\frac{5}{0} \\
: \frac{\pi}{0} \\
\frac{0}{0} \\
\frac{0}{10}\end{array}$ & $\begin{array}{l}\text { Vielfalt der Baumarten-Zusammensetzung } \\
\text { Vielfalt der Bestandsstruktur } \\
\text { Anteil heimischer, standortgerechter Baumarten } \\
\text { Totholzanteil } \\
\text { Nutzungsintensität } \\
\text { Waldrandgestaltung } \\
\text { Begehbarkeit des Bestandes } \\
\text { Dichte des Forstwegenetzes }\end{array}$ \\
\hline 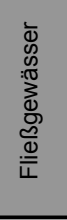 & $\begin{array}{l}\text { Gewässermorphologie } \\
\text { Ufergestaltung } \\
\text { Gewässergüte } \\
\text { Fließgewässerbreite } \\
\text { Gewässerranderschließung } \\
\text { Gewässerverbund }\end{array}$ \\
\hline $\begin{array}{l}\frac{0}{} \\
\frac{0}{0} \\
\frac{0}{d} \\
\frac{0}{0} \\
0 \\
\frac{D}{D} \\
\frac{\bar{D}}{\omega} \\
\frac{d}{\omega}\end{array}$ & $\begin{array}{l}\text { Siedlungscharakter } \\
\text { Baulicher Zustand } \\
\text { Überformung durch Baugebiete } \\
\text { Überformung im Bestand } \\
\text { Historische Gestaltungselemente } \\
\text { Ortsrandsituation } \\
\text { Gestaltung des öffentl. Raums } \\
\text { Touristische Infrastruktur }\end{array}$ \\
\hline
\end{tabular}

Eine Eigenschaft ist dann relevant, wenn von mindestens zwei der zu vergleichenden Akteure dazu Aussagen vorhanden sind und das betroffene Landschaftselement auch im Untersuchungsgebiet präsent ist, also eine Gegenüberstellung möglich ist. Die zu erstellende methodische Grundlage ist demnach nicht der Versuch eines umfassenden Modells zur systematischen Darstellung landschaftlicher Gestalt, sondern vielmehr auf die Problemstellung des Projektes zugeschnitten. Abbildung 3 erläutert die Zielstellung noch einmal.

Unter Einbeziehung der Aussagen der Akteure zur Bewertung von Ist- und Planzustand gelingt mit der Erhebung die Beantwor 


\section{Abb. 3: Gegenüberstellungen auf der Grundlage einheitlicher landschaftlicher Eigenschaften}

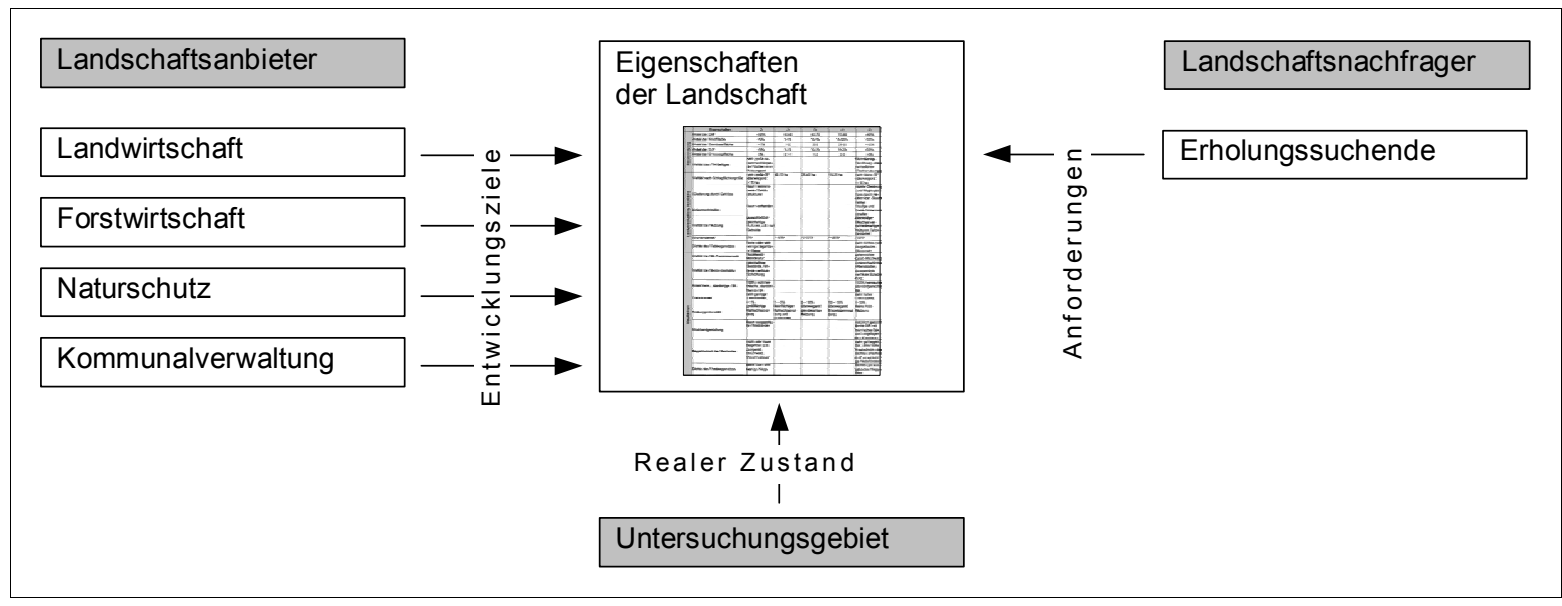

tung der forschungsleitenden Fragestellung im Hinblick auf die folgenden Aspekte.

- Wie werden die gegenwärtigen Bedürfnisse der Bevölkerung durch vorhandene räumliche Attribute erfüllt (qualitativ und quantitativ)?

- Welche Veränderungen/Tendenzen sind erkennbar?

- Welche Optionen für die Behebung von Defiziten sind denkbar?

Im Ergebnis kann aus den unterschiedlichen Anforderungen der Akteure an die landschaftlichen Parameter ein Anforderungsprofil an Landschaftsqualität entwickelt werden. Dieses Anforderungsprofil - einmal aus Sicht der Nachfrage, zum anderen aus Sicht der Gestalter - beinhaltet ein „Set“ verschiedener landschaftlicher Parameter. Diese können in Anlehnung an marketingpolitische Überlegungen als „Produkteigenschaften der Landschaft" bezeichnet werden. Der Raum wird also anhand der Elemente einer Kulturlandschaft mit klassischen Produkteigenschaften ausgestattet, die von den einzelnen Interessengruppen differenziert wahrgenommen werden, wobei die Ebenen der unterschiedlichen Interessengruppen innerhalb von Räumen in Anlehnung an eine Stakeholder-Modellierung abgegrenzt werden können.

Die landschaftlichen Parameter werden durch bestimmte Akteursgruppen gestaltet und stiften bei weiteren Akteuren einen bestimmten Nutzen. Belegbar wird das Handeln der Gestalter durch Entwicklungsziele für den Raum, der zu stiftende Nutzen durch formulierte Anforderungen an das Vorhandensein bestimmter landschaftlicher Elemente. Daher wurden im Projektverlauf Anforderungsprofile für Akteursgruppen jeweils auf Anbieter- und auf Nachfrageseite entwickelt. In erster Linie weisen diese Anforderungsprofile qualitative Aussagen zu Bedürfnissen und Wünschen an die Ausstattung der Landschaft aus, beinhalten aber auch eine Präferenzrangordnung hinsichtlich der Wichtigkeit bestimmter landschaftlicher Elemente. Die bisher erzielten Ergebnisse geben bereits Aufschluss darüber, welche Ausprägungen eines „Natur-Angebotes“ aus ökologischer und sozialer Nutzerperspektive als notwendig und sinnvoll erachtet werden können. Weiterer Forschungsbedarf ergibt sich für den Aspekt einer ökonomischen und damit monetären Bewertbarkeit der Anforderungskriterien an die Gestaltung des Raumes, die letztlich gerade für die Akteure des Angebotes besondere Relevanz besitzt.

Mit dem derzeitigen Entwicklungsstand des Modells kann jedoch bereits ein Beitrag dazu geleistet werden, Informationsdefizite über die nachhaltige ökologische Leistungsfähigkeit von konkreten Räumen zu schließen.

Weiterhin konnten Beiträge zu folgenden Forschungsaspekten erbracht werden:

- strategischem Verhalten von Akteuren in Form einer strategischen Präferenzbekundung,

- raumspezifischer Landschaftsnutzung,

- Sozialverträglichkeit der Landschaftsnutzung und gesellschaftlicher Akzeptanz, 
- Anforderungsprofilen von regionalen Akteuren sowie

- Wechselwirkungen zwischen Anforderungskriterien.

\section{Anmerkungen}

1) Ausgewählte Ergebnisse des Projektes „Qualität von Naturangeboten in urbanen Landschaften Analogien zu betriebswirtschaftlichen Ansätzen der Produktpolitik", gefördert durch das UFZ Umweltforschungszentrum Leipzig-Halle $\mathrm{GmbH}$, Teilprojekt des UFZ-Verbundvorhabens „Stadtnatur - Anforderungsprofile, Strategien und Maßnahmen zum Management von Natur in urbanen Landschaften".

2) Als Naturkapital sei hier die Gesamtheit des naturnahen Potenzials eines Raumes bezeichnet, das als öffentliches Gut angesehen werden muß und als natürlicher Ressourcenbestand eine Lebenserhaltungsfunktion besitzt; vgl. dazu u. a. die Diskussion bei Radke 1998, S. 25 ff.

3) Vgl. dazu auch die Diskussion zur „BürgerInnenbeteiligung" in Krippendorf et al. 1992, S. $42 \mathrm{ff}$.

4) Vgl. u. a. die Diskussionsbeiträge in Nutzinger 1996; hier insbesondere Gethmann, C.F.: Zur Ethik des umsichtigen Naturumgangs; Krebs, A.: „Ich würde gern mitunter aus dem Hause ein paar Bäume sehen“. Philosophische Überlegungen zum Eigenwert der Natur; Hampicke, U.: Anthropozentrik ist nicht Anthropokratie.

5) Modifizierter Lösungsansatz zur Identifikation eines Lösungskorridors in Anlehnung an Odum 1980, S. 451.

6) Eigene Zusammenstellung in Anlehnung an Krautzberger 2001, S. 130 ff. und Breuste und Moser 2000

7) Vgl. die Ergebnisse der Akteursanalyse in Henn, Patz und Streit 2002, allgemein S. 9 ff. sowie spezifisch angebots- und nachfrageorientiert S. 43-102.

8) Die Zusammenstellung des Landschaftsinventars basiert auf der Analyse von Belegquellen zur Wahrnehmung von Landschaftsbild, Nutzbarkeit der Landschaft und relevanter landschaftliche Parameter

\section{Literatur}

Agricola, S., 2001: Freizeit. Grundlagen für Planer und Manager. München, Wien: Oldenbourg

Bateman, I.J., 1995: Environmental and economic appraisal. In: O'Riordan, T. (ed.): Environmental Science for environmental Management. Harlow, UK: Longman, pp. 45-65

Breuste, J.; Moser, P., 2000: Ostdeutsche StadtUmland-Regionen unter Suburbanisierungsdruck.
Bericht zum Workshop am 4.11.1999 am UFZ Nr. $14 / 2000$

Freimann, J., 1998: Unternehmen und Stakeholder. Management zwischen Ökonomie und Politik. In: Biesecker, A., u. a. (Hrsg.): Ökonomie der Betroffenen und Mitwirkenden. Pfaffenweiler: Centaurus Verlags-Ges., S. 14-27

Henn, A.; Patz, R.; Streit, B., 2002: 1. Zwischenbericht an das UFZ, Merseburg, Februar 2002

Jänike, M., 1995: Akteure der Umweltpolitik. In: Junkernheinrich, M.; Klemmer, P.; Wagner, G.R. (Hrsg.): Handbuch zur Umweltökonomie. Berlin: Analytica

Klemmer, P., 1995: Umwelträume. In: Junkernheinrich, M.; Klemmer, P.; Wagner, G.R. (Hrsg.): Handbuch zur Umweltökonomie. Berlin: Analytica

Krautzberger, M., 2001: Nachhaltige Entwicklung und Städtebaurechtsordnung - Rio, Urban 21, Baugesetzbuch -. In: UPR Zeitschrift für Umwelt- und Planungsrecht 4, S. $130 \mathrm{ff}$.

Krippendorf, J. et al., 1992: Beim Menschen selbst ansetzen. Der Einbezug der Nachhaltigkeit in neueren Ansätzen der Regionalentwicklung in der Schweiz. In: Busch-Lüty, Ch., et al. (Hrsg.): Ökologisch nachhaltige Entwicklung von Regionen. Beiträge, Reflexionen und Nachträge. Tutzinger Tagung 1992: Sustainable Development - aber wie? Politische Ökologie 10. Jg., Sonderheft 4, S. 42 ff. Messerli, B.; Messerli, P., 1978: Touristische Entwicklung im inneralpinen Raum. Konsequenzen, Probleme, Alternativen. MAB-Mitteilungen 4, Bonn Nutzinger, H.G. (Hrsg.), 1996: Naturschutz-EthikÖkonomie: theoretische Begründungen und praktische Konsequenzen. Marburg: Metropolis-Verlag Odum, E.P., 1980: Grundlagen der Ökologie. Stuttgart, New York: Thieme, S. 451

Radke, V., 1999: Nachhaltige Entwicklung: Konzept und Indikatoren aus wirtschaftstheoretischer Sicht. Heidelberg: Physica-Verlag, S. $25 \mathrm{ff}$

Ring, I. (Hrsg.), 1997: Nachhaltige Entwicklung in Industrie- und Bergbauregionen - Eine Chance für den Südraum Leipzig? Leipzig, Stuttgart: G. Teubner Verlagsgesellschaft

\section{Kontakt}

Annette Henn

Fachhochschule Merseburg

Fachbereich Wirtschaftswissenschaften

Geusaer Str., 06217 Merseburg

Tel.: +49 (0) $3461 / 462430$

Fax: +49 (0) 3461 / 462422

E-Mail: annette.henn@ww.fh-merseburg.de

Dr. Renate Patz

Fachhochschule Merseburg

Prorektorat für Forschung und Technologietransfer 
Geusaer Str., 06217 Merseburg

Tel.: +49 (0) 3461 / 462907

Fax: +49 (0) $3461 / 462919$

E-Mail: renate.patz@ltg.fh-merseburg.de

\section{Nachhaltige Entwicklung und Innovation im Energiebereich}

\author{
von Gerd Hanekamp, Europäische Akade- \\ mie Bad Neuenahr-Ahrweiler
}

Innovationen gelten in vielen Bereichen als Vorbedingung für eine nachhaltige Entwicklung. Sie sollen einen steigenden Bedarf mit einer Reduktion des Ressourcenverbrauchs vereinbar machen. Eine nachhaltige Innovationsstrategie für den Energiebereich hat eine international besetzte Forschergruppe der Europäischen Akademie zur Erforschung von Folgen wissenschaftlich-technischer Entwicklungen Bad Neuenahr-Ahrweiler GmbH mit der Studie „Nachhaltige Entwicklung und Innovation im Energiebereich“" ausgearbeitet. Die Studie wurde im Oktober 2002 der Öffentlichkeit vorgestellt.

\section{Grundbegriffe}

\subsection{Energie}

Unter dem Energiesystem (eines Landes oder der Erde insgesamt) versteht man die gesamte Struktur der genutzten Primärenergieressourcen, der Infrastruktur zu deren Verteilung und Umwandlung in Endenergie und der spezifischen Nachfragestruktur der so genannten Energiedienstleistungen. Im Hinblick auf die Wertigkeit der Energie spielt insbesondere die Unterscheidung zwischen Bedarf an Wärme bzw. Arbeit eine Rolle, ferner die Aufteilung zwischen stationärem und mobilem Bedarf und die Rolle der Elektrizität. Angebots- und Bedarfsstruktur bestimmen zusammen das Potenzial zur Veränderung eines gegebenen Energiesystems.

\subsection{Nachhaltige Entwicklung}

Die verschiedenen Konzepte von der ,schwachen“ bis hin zur „sehr starken“ Nachhaltigkeit unterscheiden sich im Hinblick auf Substitutions- und Komplementaritätsannahmen in Bezug auf menschengemachtes Kapital und
Naturkapital. Dieser Studie liegt ein Konzept kritischer Nachhaltigkeit zugrunde, das sich auf der Grundlage des Konzepts des kritischen Naturkapitals auf wenige, aber entscheidende und in diesem Sinne kritische „Leitplanken“ oder „Engpässe“ konzentriert. Dieses Verständnis von Nachhaltigkeit schafft damit eine Verbindung zur weit entwickelten Diskussion der Setzung von Umweltstandards.

\subsection{Innovation}

Innovationen bezeichnen die Durchsetzung neuer Problemlösungen am Markt, verbunden mit neuen Faktorkombinationen. Nachhaltige Innovation bezeichnet Faktorkombinationen und neue Problemlösungen, die zu einer Senkung von Umweltbelastung und Ressourcenverbrauch führen, ohne dass dadurch Einschränkungen bei anderen gesellschaftlichen Zielen erforderlich werden. Dazu gehören nicht nur neue technologische Lösungen (Prozesse, Produkte), sondern auch neue Dienstleistungen und neue Organisationsformen.

\section{Kontext}

Veränderungen des Energiesystems müssen nicht nur einer Zielgröße - etwa der Reduktion von Treibhausgass-Emissionen - genügen, sondern einer ganzen Reihe von Zielen. Ein Zielbündel für einen nachhaltigen Umbau des Energiesystems berücksichtigt Aspekte der Ressourcenverfügbarkeit (z. B. Beschaffungssicherheit) und des Energiesystems (z. B. Verlässlichkeit und Optionsoffenheit) ebenso wie Umweltaspekte (z. B. Emissionen und Flächenverbrauch).

Die Reduzierung der $\mathrm{CO}_{2}$-Emissionen lässt sich als „Leitindikator“ für dieses Zielbündel verwenden, der einer notwendigen Bedingung entspricht und durch weitere Indikatoren (Flächenverbrauch, Optionsoffenheit u. ä.) als hinreichende Bedingungen ergänzt werden muss.

Um nachhaltige Innovation zu verstärken, sind Kenntnisse über Innovationsdeterminanten erforderlich. Ausmaß, Richtung und Geschwindigkeit der Innovationsaktivität in einer Volkswirtschaft hängen von einer Vielzahl von Faktoren ab, die zusammenfassend auch als „Nationales Innovationssystem“ bezeichnet werden und die weit über die Forschungs- und 